\title{
Linear models of two-component structure
}

\author{
Svetlana Nekrasov ${ }^{1}$, Andrew Varlamov ${ }^{1}$, Daliia Khamidulina $^{1 *}$, and Vladimir Rimshin ${ }^{2}$ \\ ${ }^{1}$ Nosov Magnitogorsk State Technical University, Department the design of structures, 455000, 38, \\ Lenin Street, Magnitogorsk, Russia \\ ${ }^{2}$ Moscow State (National Research) University of civil engineering, 129337, 26, Yaroslavskoye \\ Shosse, Moscow, Russia
}

\begin{abstract}
Two-component model of the concrete composite was considered. The model is considered in two ways. Model "matrix - defects" and model of "matrix-filler". In the model of "matrix - defects" we made the analysis of the behavior of the model depending on the number and distribution of defects in the body of the concrete prism. It is Described how to change in the number of defects in the process of loading a prismatic concrete specimen and their influence on strength and deformation properties of concrete. We obtained mathematical model of a two-component structure of concrete. We considered the changing deformation model of "matrix-filler" depending on the number and distribution of filler in the body of the concrete prism. Further models have been tested by comparison with computer models and experiments on standard prismatic specimens. It is revealed that the vertical cracks do not affect the vertical deformation of a prism.
\end{abstract}

\section{Introduction}

The analysis of models of concrete [1-5] allowed us to propose model of a simple twocomponent concrete [6,7]. For the model analysis we considered the concrete prism. We considered the location of the filler to a certain extent. The form of a filler will take the form of a ball. The analysis shows that any other form of filler is possible that will not affect the further insights [8,9]. It is assumed that the material has a fixed matrix structure (old concrete). We placed the filler in the form of balls of the same size (diameter d) in a prism $[10,11]$. This means that the sample has a wall and the filler falls only inside of the sample. We divided the sample volume into $\mathrm{n}$ parts, which can fit only two aggregate (the cell size 1,707 d). Each cell has a wall The probability of hitting one filler in one of the cells is equal to $n / n$, the second filler in the empty slot (n-1)/n and so on, the last filler in the last free cell in $1 / n$. Further, the probability that the next filler in a filled cell is changed from $\boldsymbol{n} / \boldsymbol{n}$ and on until the last possible cell to $\mathbf{1 / n}$. When filling in $\boldsymbol{m}$ cells, the probability of ingress of the filler is $\boldsymbol{m} / \boldsymbol{n}$, in this case, the probability of getting a free cell is $(\boldsymbol{n}-\boldsymbol{m}) / \boldsymbol{n}$. We took as the criterion of filling a probability of 0.5 - filling goes in the direction of greater probability of filling. When filling half of the cells, assuming that the filler in the cell is located so that it allows the second ball to be located in it, we get $50 \%$ of the voids among

\footnotetext{
*Corresponding author: loza mgn@mail.ru
} 
the filled cells and the probability of the next ball getting into a free cell is determined by $0.5 /(0.5+0,25)=0.67$. Therefore, free cells continue to fill up before occupying balls with $67 \%$ of them. After this, free and filled cells with one ball start to fill up, moreover, cells filled with one ball fill up the second faster than free cells with the first, up to filling the entire field with balls. However, this case of filling is unlikely, since the hit of the ball in the cell so that there is free space for getting into the cell of the second ball is small. To simplify the calculations, we divide the cell into additional cells with a side of $0.05 \mathrm{~d}$ and suppose that the center of the ball can only be in one of these cells, then the probability of the second ball getting into this cell is 0.005 , and the probability of filling all empty cells at first increases to $99 \%$.

Similarly, when dividing the total area (flat section) into cells along four balls (cell size $2 \mathrm{~d}$ ), when accepting the conditions for dividing a cell into cells: the probability of filling such a cell with one ball -1 , two balls 0.044 ; three balls - 0,00081 (which is already unlikely); four balls - 0.00000145 . Increasing the grain expansion (reducing the filler diameter to 0.95 from the original size, leading to an increase in the amount of the solution part by 1.36 times) increases the likelihood of hitting two balls to 0.097 , four to 0.000256 .

When selecting such an artificial material as concrete, a structure is created, the amount of aggregate and mortar is dosed. In Fig. 1, a. the square of a unit area is shown, in which two grains of filler are in contact with each other. Inside the square, the area equal to the probability of two contacting grains falling into the square is shown. Obviously, with a decrease in the diameter of the grains, the probability of them falling into this square increases, and an increase in the distance between the grains decreases the probability of falling into the square. Therefore, when freely squared, it is most likely that the grains will form heaps. In contrast to this case, when the filler is mixed in the matrix of artificial material, they try to reduce the randomness of the arrangement, the energy is spent on reducing the entropy and structuring the material. In this case, the location of the aggregate occurs uniformly in volume. Then, dividing the sample volume into smaller equal volumes, they should receive the same amount of aggregate in these smaller volumes. For example, dividing a rectangular plate into two identical parts vertically, then the resulting parts into two identical parts along the horizontal, etc. : we get at the end of the division the smallest cell in which the uniformity of volumes is preserved (Fig. 1, b). However, in this case, the previous cell must be symmetrical about one of the separation axes.
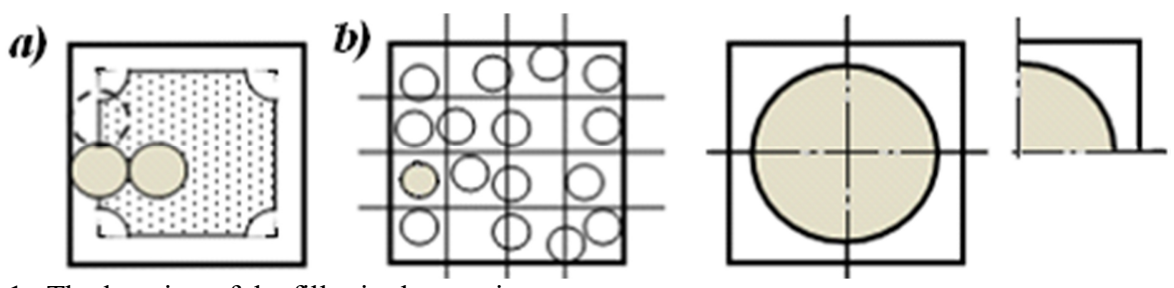

Fig. 1. The location of the filler in the matrix

a) contact grain filler; b) divided grain filler.

Therefore, a perfectly uniform distribution should be symmetrical with respect to the fission axes, and in the case of volume fission, fission planes.

As a result of a statistical analysis of the aggregate distribution model in artificial material, we can state:

- unlike statistically chaotic filling of the mixture volume, artificial materials are created to the desired structure and the aggregate is forced to be placed in a volume determined by it, where it is already shifted in accordance with random processes.

- an ideally homogeneous model is symmetrical with respect to the selected axes; 
- there is a minimal cell in an ideal model, dividing which we get a cell with an inhomogeneous distribution of material.

- in a real model, two types of inhomogeneities must be considered - heterogeneity between cells and heterogeneity inside the cell.

\section{The simplest models}

Certainly, at the moment [12-17], it is impossible to describe the stress - strain - time temperature (defining equations) real concrete relationships with simple formulas, therefore, it is necessary to obtain simple physical equations by idealizing its behavior. The simplest models of dissimilar materials [18] are shown in Fig. 2 and Fig. 3. The presented models are two-factor: aggregate and matrix. As a filler, it is possible to consider either a real filler having a corresponding deformation modulus or matrix defects for which we take the modulus equal to zero.

On the models shown in Fig.2.3, the graphs of the $\varepsilon-\sigma$ dependence are plotted in relative units for the materials making up the model (matrix and aggregate). The intermediate graphs of the models are constructed depending on the ratio of the number of constituent materials $(1: 0.5 ; 1: 1 ; 1: 2)$. For the circuit (Fig. 2), all graphs have the form shown in Fig. 2 , b. The heterogeneity scheme in Fig. 2, b does not contribute to the redistribution of stresses and strains and therefore cannot be taken as a basis for modeling two-component concrete. On the other hand, the elements of the circuit in Fig. 2, b can occur in real concrete.
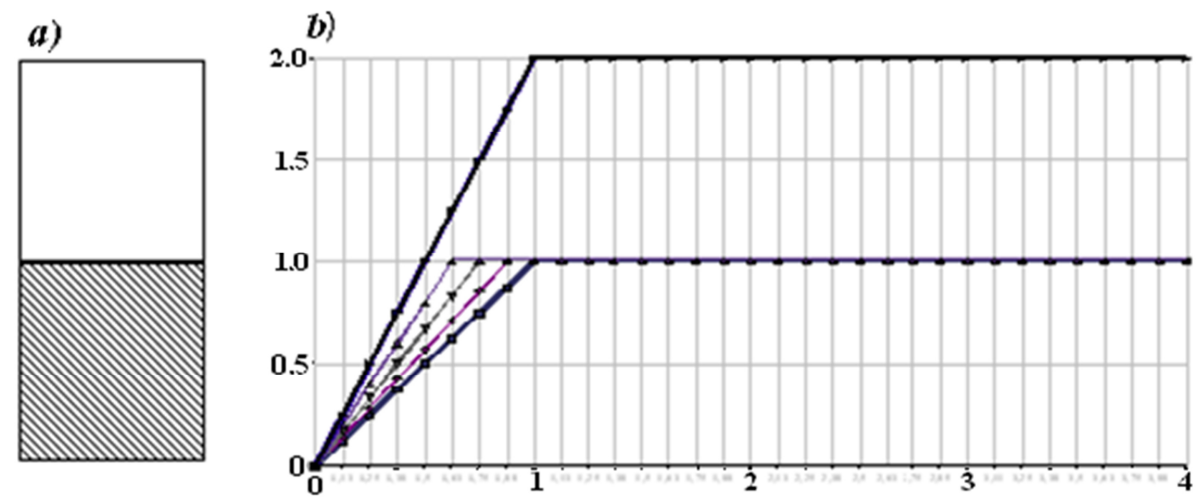

Fig. 2. A horizontal arrangement of the filler in the sample

a) scheme; $\boldsymbol{b}$ ) charts " $\boldsymbol{\varepsilon}-\boldsymbol{\sigma}$ " for given schema.
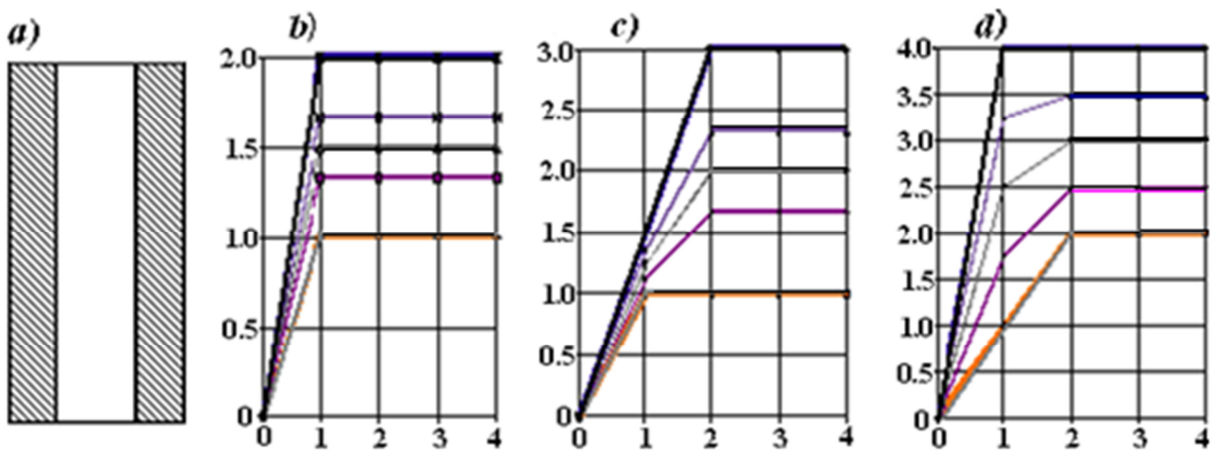

Fig. 3. The vertical arrangement of the filler in the sample 
a) scheme; $\boldsymbol{b}, \boldsymbol{c}, \boldsymbol{d})$ charts " $\varepsilon-\sigma$ " for given schema

For "roughly" inhomogeneous materials, such as concrete, the use of the above schemes (Fig. 2,3) is not rational, since it does not allow taking into account the inhomogeneity of stresses and deformations. But it should be noted here that we are talking about certain sizes of elements and their ratio. It is possible to speak of deformations of concrete as a material with a sufficient averaging of deformations.

For example, by measuring strains on a base equal to the size of the aggregate, we obtain the magnitudes of the strains noticeably depending on the location of this base relative to the aggregate. The element to be distinguished must be large enough so that the average displacement gradient can be determined, but also small enough so that it does not have jumps in the displacement gradient. For example, we divide the prism into separate inhomogeneous parts (it has already been noted that we will use two components in all considered models).

\section{Distributed model}

In Fig.4 shows a schematic breakdown of homogeneous microsatellite or defects in the concrete prism, the component binding (in the diagram, the light and having a larger volume), we call the matrix, the dark color shows the filler or defects. In the diagram, Fig.4, a rough breakdown on heterogeneous components is shown, which is more in keeping with the structure of ordinary concrete. In the diagram, Fig.4, used heterogeneous components statistically evenly spaced around the volume of the prism. The diagram in Fig.4,b present in a modified form diagrams in Fig. 4,c - 4,f. In the diagram, Fig.4,c is the particle aggregates parallel offset horizontally to the right side of the prism, the diagram Fig.4, $\boldsymbol{d}$ the ranking of particle diagrams in Fig.4,c. In the diagram, Fig.4,e all the particles of the filler parallel to the vertically offset to the lower side of the prism, the diagram Fig. $4, f-$ were ranked particle diagrams in Fig.4,e. The distribution of the filler shown in Fig.4, $\boldsymbol{d}$ and $4 \boldsymbol{f}$ corresponds to the statistical distribution curve of the filler in the matrix.
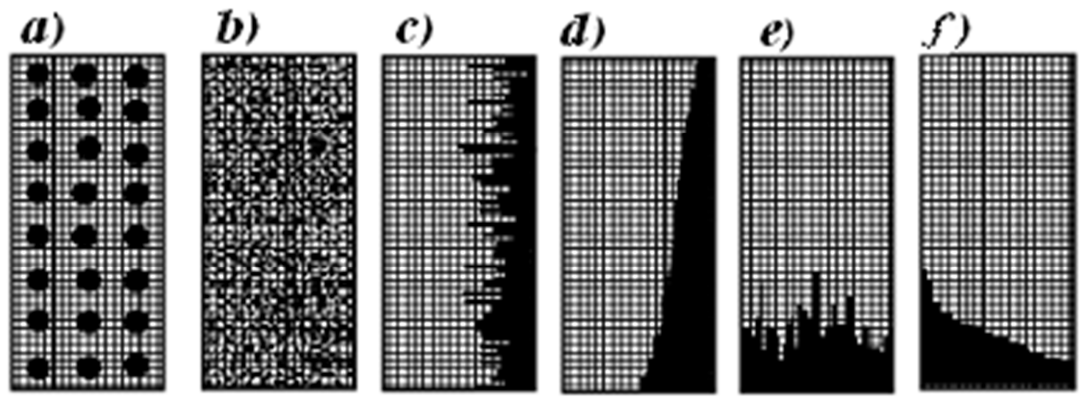

Fig. 4. The distribution of structural defects in the prism

Three schemes are distinguished from the considered ones: equally distributed (Fig. 4, b), a matrix with the inclusion of a filler (Fig. 4, a), and coarse (Fig. 2). The first scheme equally distributed (Fig. 4, b) is characteristic of the mortar part of concrete, while if we take a two-linear scheme of the material, then an equally distributed scheme when analyzing the material's work for longitudinal deformations can be represented in a simplified In the general form shown in Fig. 4, c as for the average distribution of the material or as a diagram in Fig. 4, $\mathrm{d}$ as for the statistical distribution of the material along the height of the prism. Schemes can also be considered as defect schemes in a homogeneous material. The second scheme (Fig. 4, a) is characteristic of concrete and, in 
contrast to the scheme of Fig. 3, not only the amount of aggregate, but also its size and the distribution of aggregate in the volume have a significant influence on its behavior. how and in what form the particles are concentrated. The third scheme (Fig. 4, b) is most likely useful in analyzing the movement of a material in time (fluidity).

Based on the foregoing, it is proposed to use the third scheme to describe the behavior of concrete during plastic deformation. Here, the results of modeling crack growth in a concrete prism [6] are taken into account, which showed a slight effect on the vertical longitudinal deformations of concrete of vertical crack-like defects. The behavior of concrete (mainly a change in the modulus of elasticity) is described by the second scheme. Use the first scheme to describe the operation of the soluble concrete part and use the results obtained hereinafter to describe the matrix behavior in the second scheme. In the first scheme, the distribution of aggregate does not play a significant role, therefore, in a further analysis of the behavior of the material according to this scheme, we use it as flat.

\section{The estimated model}

Let us consider the simplest design scheme of the distribution of defects is shown in Fig.5.
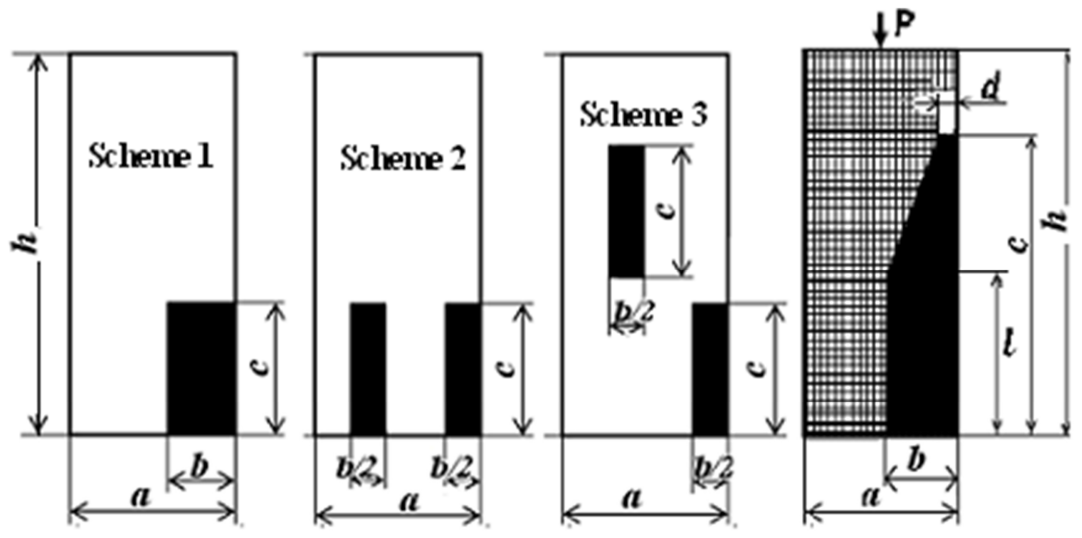

Fig. 5. The design scheme of the distribution of defects in the prism

The dark area in the presented diagrams show material that do not resist external impact. The material of the light zone operates elastically (module E). The light material does not change its properties depending on external influences. Consider flat the prism is loaded by the force $\mathbf{P}$. Write the formula of deformation for the prisms:

Scheme 1 and Scheme 2:

$$
\Delta l=(h-c) \frac{p}{a \bar{E}}+c \frac{p}{(a-b) \bar{E}}=\frac{p h}{\bar{E}} \frac{1+c_{/ h}{ }^{/} / a^{0 / a}}{a-b}=\frac{p h}{E a}\left(1+\frac{c_{h} D_{a}}{1-b / a}\right) .
$$

Scheme 3:

$$
\Delta l=\frac{P h}{E} \frac{1+c_{h}{ }^{0 / a}-0 / 2 a}{a-b / 2}=\frac{P h}{E a}\left(1+\frac{c_{h}{ }^{0 / a}}{1-b / 2 a}\right) .
$$

Similarly, you can divide the dark zone bxc into another number of elements, preserving its area, while the nature of the obtained dependencies will not change. An analysis based on the obtained dependences showed that an increase in the dark zone in height (an increase in the factor before a in the denominator in the expression according to Scheme 3) reduces strains and thereby the energy consumption for compressing the prism. This allows us to draw 
the following conclusion: in a homogeneous material, the direction of growth of the fracture zones (dark zone) goes in the direction of the direction of deformation, and only then in the direction of its expansion. A similar conclusion is given by the study of the expansion of the dark zone. External energy is expended on the growth of the defect zone. With an increase in the size of the dark zone of the HCF, strains increase. Therefore, as the number of defects increases, the energy consumption for their further growth also increases [7]. Or in another way - with an increase in energy consumption for plastic deformations, the costs of elastic deformation of a prism increase until elastic deformations reach a limiting value. It should be noted here that the above conclusions relate to the schemes under consideration.

Another conclusion from the analyzed schemes is that the elastic deformations of a prism depend not only on the number of defects, but also on their distribution. Therefore, for further analysis, we write down the prism deformations for the trapezoidal design scheme shown in Fig. 5.

$$
\delta=\frac{\sigma h}{E}\left(1+\frac{c / h}{2 a / d+b-1}-l / h \frac{d-b}{h(a-b)(2-d+b / a)}\right)
$$

Rewrite the last equation:

$$
\frac{\delta E}{\sigma h}=\frac{E_{0}}{E^{\prime}}=\left(1+\frac{c / h}{2 a / d+b-1}\right) .
$$

As can be seen from the dependencies factors was divided: on directly related to properties of materials (left part of the formula) and structure factors (right part of formula (4)). The ratio of initial modulus of elasticity of the material $\left(\boldsymbol{E}_{0}\right)$ to secant modulus $\left(\boldsymbol{E}^{\prime}\right)$ is defined only the structural parameters of the model. This means that in the initial state the material has no defects (black areas) in this case, deformation of the prism will be determined only by the modulus of the matrix $\boldsymbol{E}=\boldsymbol{E}_{\boldsymbol{0}}$.

The results show that when $\mathrm{b} / \mathrm{a} \rightarrow 0$, we obtain crack-like defects which not affect longitudinal deformation of the prism. If the prism has defects the same width $\mathrm{b} / \mathrm{a}=$ const, the first will begin to grow shorter defect (in this scheme it is not considered transverse deformation of the elements). Given the previous analysis for the considered model we obtain: if at the beginning of the loading we had a triangular area of defects, then it gradually goes into the trapezius and then to rectangular. After that, the rectangular area is expanded to achieve a non-defective area of the ultimate strain. For old concrete (long working under axial load) it is possible to initially assume a rectangular plot of defects. Demonstration of such a transition are shown in Fig.6.
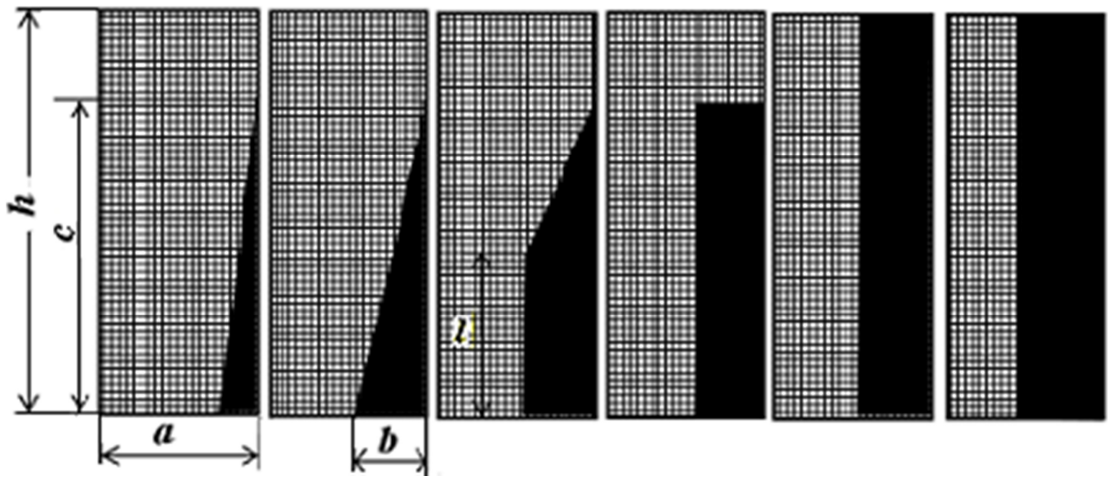

Fig. 6. The growth of structural defects (left to right) 
Consider the table.6.11[SP 63.13330.2018 Concrete and reinforced Concrete structures. The basic provisions]. The data of this table will move in the first two rows of the table.1.( $\left(\mathrm{E}_{\mathrm{b}}\right.$, in $\left.\mathrm{MPa} 10^{3}\right)$. The third line is placed relative modulus of elasticity of concrete. Relative module adopted in relation to the base B100.

Table 1. The results of the comparison

\begin{tabular}{|lcccccccccccccccc|}
\hline The concrete class & 3,5 & 5 & 7,5 & 10 & 15 & 25 & 30 & 35 & 40 & 50 & 55 & 60 & 70 & 80 & 90 & $\mathbf{1 0 0}$ \\
\hline The modulus of elasticity & 9.5 & 13.0 & 16.0 & 19,0 & 24,0 & 30,0 & 32,5 & 34,5 & 36,0 & 38,0 & 39,0 & 39,5 & 41.0 & 42.0 & 42.5 & $\mathbf{4 3}$ \\
The relative module & 4.52 & 3.31 & 2.69 & 2.26 & 1.79 & 1.43 & 1.32 & 1.25 & 1.19 & 1.13 & 1.10 & 1.09 & 1.05 & 1.02 & 1.01 & $\mathbf{1}$ \\
$\mathbf{b} / \boldsymbol{a}$ (Fig. 5) & 2 & 3 & 4 & 5 & 6 & 8 & 9 & 10 & 11 & 14 & 15 & 18 & 20 & 25 & 50 & $\mathbf{1 0 0}$ \\
$E_{0} / E^{\prime}$ rectangle & 2 & 1.5 & 1.33 & 1.25 & 1.2 & 1.14 & 1.12 & 1.11 & 1.10 & 1.08 & 1,07 & 1.06 & 1,05 & 1,04 & 1.02 & $\mathbf{1 . 0 1}$ \\
$E_{0} / E^{\prime}$ triangle & 1.33 & 1,2 & 1,14 & 1,11 & 1,09 & 1,07 & 1,06 & 1,05 & 1.05 & 1.04 & 1.03 & 1.03 & 1.03 & 1,02 & 1.01 & $\mathbf{1 . 0 0}$ \\
\hline
\end{tabular}

We analyzed the table as for the prisms of homogeneous material having a different number of defects and, accordingly, different strength. For concrete B100 will assume defectfree structure $\left(\boldsymbol{E}_{0} / \boldsymbol{E}^{\prime}=1\right)$. For further analysis it is not essential, knowing that the initial modulus of elasticity of the concrete at the further increase of its class, is increasing slightly. For the weak concrete will take the rectangular shape of the defects (as for the section that is most weakened by the defects). Then he $\frac{E_{0}}{E^{\prime}}=1+\frac{1}{a / b-1}$, hence we obtain $a / b \approx 2$. Considering further the rectangular shape of the defect will receive, respectively, the number of values that are listed in the table.1. For concrete with a triangular area of defects дефектов $\frac{E_{0}}{E^{\prime}}=1+\frac{1}{2 a / b-1}$ respectively receive the row values, also shown in the last row of the table. 1 . Comparison of the results was obtained with the ratios of modules for concrete given in the table, to some extent confirms the transition from a triangular (or close to it) area to the rectangular (or close to it). There are three areas: - high-strength concrete the concrete of classes B70 and above the transition zone B70 - B50, concretes of medium strength B50 $\mathrm{B} 15$, and concretes with complex structure of defects is less than B15.

Next, we considered the change of modulus of deformation for the sequence change of the defects in the schemes of Fig.6. Overall, the relative volume of defects in the $\Omega$ is increased from left to right in sequence $0,08 \boldsymbol{a h} ; 0,16 \boldsymbol{a h} ; 0,24 \boldsymbol{a h} ; 0,32 \boldsymbol{a h} ; 0,4 \boldsymbol{a h} ; 0,48 \boldsymbol{a h}$.

For each scheme we write equation based on (1) left to right:

$$
\begin{aligned}
& \frac{E_{0}}{E^{\prime}}=1+\frac{0,8}{2 \times 5-1}=1,089 ; \quad \frac{E_{0}}{E^{\prime}}=1+\frac{0,8}{2 \times 2,5-1}=1,2 ; \\
& \frac{E_{0}}{E^{\prime}}=1+\frac{0,8}{2 \times 2,5-1}-0,4 \frac{-0,4}{(0,6)(2-0,4)}=1,367 ; \quad \frac{E_{0}}{E^{\prime}}=1+\frac{0,8}{2 \times 1,25-1}=1,53 ; \\
& \frac{E_{0}}{E^{\prime}}=1+\frac{1}{2 \times 1,25-1}=1,667 ; \quad \frac{E_{0}}{E^{\prime}}=1+\frac{1}{2 \times 1,042-1}=1,923
\end{aligned}
$$

and for $\boldsymbol{\Omega}=0,56 \boldsymbol{a h}: \frac{E_{0}}{E^{\prime}}=1+\frac{1}{2 \times 0,893-1}=2,272$.

Graphics obtained with the use of the calculations shown in Fig. 7. 


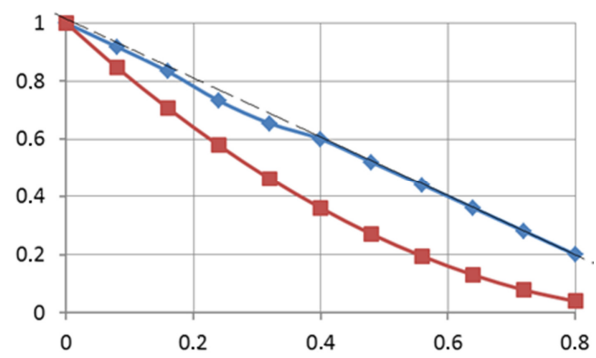

Fig. 7. The change in the strength and deformability of the model from the relative number of defects.

On the horizontal axis Fig.7. - the relative number of defects in the model $\boldsymbol{\Omega}$. Vertical axis - relative units. Upper panel - the relative change of the initial modulus of deformation of the model $\boldsymbol{R}_{0} / \boldsymbol{R}^{\prime}$. Bottom graph is the relative change in the strength of the model . The dependence of the relative change in the strength of the model obtained on the basis of the Feret formula:

$$
\frac{R_{0}}{R^{\prime}}=\frac{R_{\Pi}}{R_{M}}=\frac{\left(\alpha d_{0}^{n}\right)_{\Pi}}{\left(\alpha d^{\prime 2}\right)_{M}}=\frac{\alpha\left((1-\Omega)^{2}\right)}{\alpha\left(1^{2}\right)}=(1-\Omega)^{2} .
$$

When you use the formula Feret assumed that the coefficient $\boldsymbol{\alpha}$ is the same for all the investigated range (for same material), the ratio $\boldsymbol{n}=2$ is adopted as proposed Feret. According to the above graphs show that the deformation modulus of the model depending on the number of defects varies almost linearly. A slight deviation from linear dependence (deviation at $\boldsymbol{\Omega}=0,24$ is of $0.732 / 0,74=0,989$ or less than $1.1 \%$ ) is in a trapezoidal plot defects. However, judging by the graph, the relationship of deformation and the number of defects can be taken linear, regardless of the plot flaws. View graphics obtained $\boldsymbol{E}_{\boldsymbol{d}} / \boldsymbol{E}^{\prime}=1-$ $\boldsymbol{\Omega}$. Consideration of the two-component model shows that the change of the elastic modulus [6] is a direct measure of the increase in the number of defects or decrease the expected strength of the material.

For the circuit in Fig.7 write expressions similar to (1)-(2) for samples with different elastic module of dark and light zones. For light zone $\boldsymbol{E}_{\boldsymbol{M}}$ (the matrix), dark zone $\boldsymbol{E}_{3}$ (filler). Module for prism will be denoted by $\boldsymbol{E}_{\boldsymbol{I}}$.

General view of the dependence:

$$
\frac{E_{M}}{E_{\Pi}}=1-c / h+\frac{2(c / h-l / h)}{2+\left(E_{3} / E_{M}-1\right)(d / a+b / a)}+\frac{l / h}{1+\left(E_{3} / E_{M}-1\right) b / a},
$$

for uniform distribution материала $\boldsymbol{d}=\boldsymbol{b}$

$$
\frac{E_{M}}{E_{\Pi}}=\frac{1}{1+\left(E_{3} / E_{M}-1\right)^{b / a}} \text {. }
$$

The above dependences reflect the effect on the deformation modulus of the material of the heterogeneity of its structure and the properties of its structural components. As simplification schemes are simplified and the resulting dependencies.

Let us consider the dependences for the schemes shown in Fig. 4, a. and fig. 8. In the diagrams of Fig. 8, the diameter of the filler increases from left to right while maintaining its total share in the volume of the prism. 

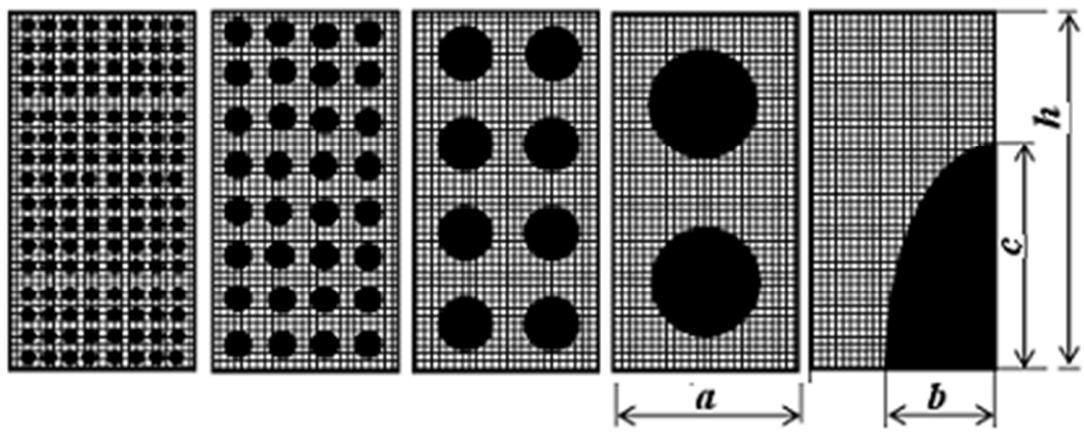

Fig. 8. The design scheme for spherical filler

In the case of homogeneous deformations (no stress concentration) and elastic behavior of the materials of the circuit shown in Fig.8, give the same expression (5).

$$
\begin{aligned}
\frac{E_{M}}{E_{\Pi}} & =1-c / h\left[1-\frac{1}{1+\pi / 4(b / a)\left(E_{\left.3 / E_{M}-1\right)}\right.}\right] \\
\text { or } \quad \frac{E_{M}}{E_{\Pi}} & =1-2 \sqrt{\frac{\theta}{\pi}}\left[1-\frac{1}{1+\sqrt{\frac{\theta \pi}{4}\left(E_{3 / E_{M}}-1\right)}}\right],
\end{aligned}
$$

where $\boldsymbol{\theta}$ is the relative area of the filler in the cross section.

In the expression (4) with $\boldsymbol{c}=\boldsymbol{n d}, \boldsymbol{b}=\boldsymbol{m} \boldsymbol{d}$, where $\mathrm{n}$ and $\mathrm{m}$ are the number of filler width $\boldsymbol{b}$ and the height of the prism $\boldsymbol{h}$, respectively, $\boldsymbol{d}$-diameter of the filler

\section{Conclusions}

By a similar principle, flat and three-dimensional models of concrete with spherical and ellipsoidal aggregates were built ( 8 models). Models were tested by comparison with computer models and experiments on standard prismatic samples [9]. Model (4) showed some of the best results. Consideration of a flat model is also explained by a simpler determination of the size and amount of aggregate by material cut. Providing uniform prism deformation (for example, when testing a prism in a piston unit), formula (4) is also valid for a roughly heterogeneous distribution of aggregate.

All schemes show the absence of the effect of crack-like vertical defects on the deformation of prisms.

\section{References}

1. E.Kuzina, V.Rimshin, V. Kurbatov, IOP Conference Series: Materials Science and Engineering, 463(4),042009 (2018)

2. A.A.Varlamov, V.I.Rimshin, S.Y.Tverskoi, IOP Conference Series: Materials Science and Engineering, 463(2),022028 (2018)

3. N.I. Karpenko, V.A. Eryshev, V.I. Rimshin, IOP Conference Series: Materials Science and Engineering, 463(3),032024 (2018)

4. A.A.Varlamov, V.I. Rimshin, S.Y. Tverskoi, Materials Science Forum, 931 340$345(2018)$ 
5. Y.M. Bazhenov, V.T. Erofeev, V.I. Rimshin, S.V. Markov, V.L. Kurbatov, Engineering Solid Mechanics, 4(4), 219-225 (2016)

6. A.A. Varlamov, KGASU, 29, 19-26 (2014)

7. V.I. Rimshin, A.A. Varlamov, Textile technology series, 3, 63-68 (2018)

8. Y.M. Bazhenov, V.T. Erofeev, V.I. Rimshin, S.V. Markov, V.L. Kurbatov, Engineering Solid Mechanics, 4(4), 219-225 (2016)

9. A.A.Varlamov, V.I. Rimshin, S.Y. Tverskoi, IFAC-PapersOnLine, 51(30), 808$811(2018)$

10. A.A.Varlamov, V.I.Rimshin, S.Y.Tverskoi, IOP Conference Series: Materials Science and Engineering, 463(2), 022029 (2018)

11. E. Kuzina, A. Cherkas, V. Rimshin, IOP Conference Series: Materials Science and Engineering, 365(3),032053 (2018)

12. V.M. Bondarenko, A.M. Kurzanov, V.I. Rimshin, Bulletin of the Russian Academy of Sciences , 70(11), 1005-1009 (2000)

13. I.N. Akhverdov, Foundations of physics Concrete ( Stroiizdat, Moscow ,1981)

14. V.I. Rimshin, E.A. Larionov, N.T. Vasylkova, Structural mechanics of engineering structures and structures, 2, 77-81 (2012)

15. K.A. Piradov, K.A. Guzeev, Concrete and reinforced concrete, 5 ,17 (1998)

16. A.A. Varlamov, Concrete and reinforced concrete, 3, 27-30 (2012)

17. K.Z. Galustov Concrete and reinforced concrete, 5, 11-15 (2008)

18. Y. M. Bazhenov, Technology of concrete ,415 (1987) 